\title{
Building a foundation for evidence-based practice: experiences in a tertiary hospital
}

The aim of this article is to report on the creation and early innovations of an evidence-based nursing committee in a tertiary hospital. During a post-merger transitional period, the Evidencebased Nursing (EBN) Committee conducted a number of projects, which included selecting an evidence-based practice (EBP) model for nursing; developing a process for prioritising and disseminating research findings to healthcare programmes; and evaluating an important EBP application in direct patient care.

Hamilton Health Sciences (HHS), a 1157 bed tertiary hospital providing inpatient and outpatient acute and long term care across several health programmes, is a recent merger of 2 hospitals at 4 sites in a large city in southern Ontario, Canada. Approximately 2900 nurses (2550 Registered Nurses and 380 Registered Nursing Assistants) are employed by HHS in either full or part time positions in health programmes that range from infertility services and obstetrical care to a large and progressive cardiac surgery programme. HHS provides regional services to 2.2 million people in Hamilton and Central South Ontario.

In 2000, the Nursing Practice Committee (NPC), a nursing body that represents direct care nurses, sets the general direction for nursing, and is accountable to the Chief Nursing Officer, identified 3 priorities for development. One of these priorities was to develop the use of EBP by nurses at the bedside. Therefore, with accountability to the NPC, the EBN Committee was created and given the mandate of developing the use of EBP at the patient care level and recommending processes that would promote EBP among direct care nurses. In 2002, the mandate and responsibilities were expanded (see table 1).

Under the co-leadership of a clinical nurse specialist (BK) and a school of nursing faculty member with a track record in health services research (EAM), the EBN Committee membership was established taking the following factors into account:

- hospital sites

- healthcare programmes

- major representation of direct care Registered Nurses and Registered Practical Nurses

- academic and research representation from McMaster University School of Nursing

- links to community care

Table 1 Terms of reference for Hamilton Health Sciences (HHS) Evidence-Based Nursing (EBN) Committee

\section{Mission}

- To foster evidence-based practice among HHS nurses Responsibilities

- To advance knowledge about evidence-based practice

- To make recommendations about processes that promote evidence-based practice to appropriate decision making bodies

- To facilitate the appropriate application of best practice guidelines and other evidence-based findings to practice

- To develop communication mechanisms that inform nurses about evidence-based practices

- To collaborate with others to evaluate evidence-based trends in practice

- To provide nursing students with an opportunity to learn about the EBN Committee and the implementation of evidence-based practice in nursing
Two hour EBN Committee meetings were scheduled monthly after NPC meetings for the convenience of members serving on both committees. At the outset, funds were provided for direct nursing staff replacement costs, so that EBN Committee members could complete preparatory work and attend meetings. In the spring of 2002, replacement costs stopped because of fiscal cutbacks to Ontario hospitals.

From the beginning, several barriers to the implementation of EBP by nurses were recognised and taken into account when planning change. ${ }^{1}$ Firstly, direct care nurses on inpatient units have little control over their workloads, which can hamper involvement in non-direct care activities, such as learning about EBP or participating in evidence-based clinical projects. ${ }^{2}$ Compounding this, a continuing nursing shortage resulted in the reliance on many overtime nursing hours.

The merger of 4 different healthcare facilities brought differing cultures and organisational challenges to the fledgling, albeit large, hospital. Furthermore, the merger itself created an imperative of forging a unified organisation, and numerous merger goals became priorities for action. New organisational priorities, such as re-developing organisational structures that were no longer suitable after the merger, superseded those of the nursing staff so that goals, such as the promotion of EBP by nurses, were placed on hold. Another barrier to the implementation of EBP was the limited access to research journals and other research resources within or close to the clinical areas of the hospitals. Even if research resources are readily available, as Funk et al found, most nurses lack the training and skills in effective literature searching and critical appraisal. ${ }^{1}$ Furthermore, experiential knowledge is often favoured by nurses over empirical evidence. ${ }^{3}$ A critical issue, identified by Newman and Papadopoulos, is lack of support by ward managers for the concept or application of EBP. ${ }^{4}$ Some HHS nursing staff reported a lack of support, although infrequently. In these cases, nursing staff reported that information seeking and critical appraisal are not valued as a part of the nursing culture on the unit, and limited access to computer resources exacerbates the problem. Communication with nursing staff across the 4 sites was problematic because of the lack of a common electronic communication system and incomplete electronic address listings for nursing staff. Finally, the complexities of modern healthcare organisations can create a formidable barrier to creating change in clinical practice. The sound administration of today's healthcare organisation depends on the interrelatedness of administrative bodies within the agency. This interrelatedness creates professional interdependencies that require considerable communication, negotiation, and collaboration and can result in protracted change processes that are both energy and time consuming.

Despite these barriers, several opportunities for change were identified:

- the nursing organisation had identified EBP as a priority for planning and action

- the EBN Committee volunteers were well motivated to learn about EBP and effect change 
- at an early stage, the EBN Committee viewed Evidence-Based Nursing, the abstract journal, as an important resource for research dissemination ${ }^{5-6}$

\section{Selecting an EBP model for nursing}

As a first step in developing a foundation for EBP by nurses at HHS, the EBN Committee conducted a project to (i) search, retrieve, and synthesise the current literature describing EBP models to help staff nurses use EBP concepts and apply them in clinical practice, and (ii) recommend the adoption of a specific EBP model for use by HHS nurses. Literature searches of several databases, including CINAHL and Medline, were done using terms such as "diffusion of innovation" and "evidence-based medicine." Other search techniques included reviewing references lists and internet sources and consulting with the Canadian Centre for EvidenceBased Nursing. ${ }^{7}$ From these sources, 78 EBP model related titles were retrieved, and 23 distinct models were identified.

Five criteria for relevance to clinical nursing in tertiary care hospitals were established based on HHS needs and those cited in the literature. Firstly, the model had to be clear and concise so that diagrammatic representation would allow quick assimilation of the concepts and organisation of steps. Secondly, it required comprehensiveness from the beginning stages through to implementation and evaluation steps. Thirdly, ease of use when applying the concepts to direct healthcare issues was needed. Fourthly, the model had to be generalisable to varied HHS healthcare programmes. Finally, published evidence of the model's evaluation in practice was deemed a strength of the model.

Each model was rated independently by 4 people (EAM, BK, an emergency room nurse, and an occupational health nurse). Each criterion was scored on a $0-5$ point scale $(0=$ lowest rating, $5=$ highest rating). Data were entered and analysed using SPSS. Total rating scores, mean rating scores, and standard deviations were generated for each model. The preferred implementation model was selected based on the highest mean scores for the total rating of each model. The data were re-analysed excluding the fourth and fifth criteria, as few empirical studies of the models' effectiveness were identified in the published literature and little variation in the scoring of the generalisability criterion was noted in the ratings. Table 2 shows the results for the top 8 rated models in the analyses, ${ }^{8-15}$ with the mean total scores for the 5 criteria and the 3 core criteria (clear and concise, comprehensive, and ease of use by direct care nurses).

Examining the mean total scores, using either set of criteria, the mean total rating score for the Rosswurm and Larrabee model was well above those of the other models. ${ }^{8}$ Only one minor difference in the 2 sets of rankings was found: the comparison showed a reversal in the rank order of the Aurora and Stetler models. ${ }^{9-10}$ The analyses enabled a clear decision to adopt the Rosswurm and Larrabee model. ${ }^{8}$

Table 2 Ratings for the top 8 evidence-based practice implementation models

\begin{tabular}{|c|c|c|c|}
\hline \multicolumn{2}{|c|}{ Total rating for 5 criteria* } & \multicolumn{2}{|c|}{ Total rating for 3 core criteria* $\dagger$} \\
\hline Model & Mean (SD) & Model & Mean (SD) \\
\hline Rosswurm and Larrabee ${ }^{8}$ & $20.00(3.74)$ & Rosswurm and Larrabee ${ }^{8}$ & $13.25(1.26)$ \\
\hline lowa $^{11}$ & $16.75(6.02)$ & lowa $^{11}$ & $10.25(3.77)$ \\
\hline Children's Hospital of Philadelphia ${ }^{12}$ & $16.25(6.80)$ & Children's Hospital of Philadelphia ${ }^{12}$ & $9.25(4.50)$ \\
\hline Stetler $^{10}$ & $16.25(2.22)$ & Aurora $^{9}$ & $8.80(2.05)$ \\
\hline Aurora $^{9}$ & $15.20(2.78)$ & Stetler ${ }^{10}$ & $8.75(1.26)$ \\
\hline Diffusion of Innovation $^{13}$ & $14.00(8.79)$ & Diffusion of Innovation $^{13}$ & $8.75(4.99)$ \\
\hline Research Nurse Intern Program ${ }^{14}$ & $14.00(7.35)$ & Research Nurse Intern Program ${ }^{14}$ & $8.75(4.27)$ \\
\hline Process of Research Utilization ${ }^{15}$ & $12.75(2.63)$ & Process of Research Utilization ${ }^{15}$ & $8.75(1.70)$ \\
\hline
\end{tabular}

${ }^{*}$ Highest possible total mean for the 5 criteria and 3 criteria ratings is 25 and 15 , respectively.

†Core criteria: clear and concise; comprehensive; ease of use by clinicians.
The EBN Committee made several adaptations to the framework to better reflect the needs of HHS nurses and the context in which the model would be used by nurses in the organisation. so that the model is viewed as a cycle (figure 1). This emphasises the need for continuing examination of clinical questions over time and permits application of the EBP process or examination of projects at discrete steps in the cycle. When data are insufficient to support a decision about whether to maintain a specific practice or adopt a new one, the added step, "stimulate inquiry," encourages nurses to seek out colleagues, such as nurses with advanced research training, to assist in developing an answerable research question and possibly lead a research project. In its current form, this model is dynamic, iterative, and clinician friendly in that it mirrors the nursing process; is clear, concise, and easily depicted as a diagram; and is applicable to clinical care. The model for EBP is being used by the EBN Committee to focus its work and to orient staff nurses to the EBP concept and the sequence of steps used to implement it. For dissemination purposes, the model is incorporated into posters and pamphlets to reinforce EBP concepts.

Hamilton Health Sciences nursing model for implementing evidence-based practice

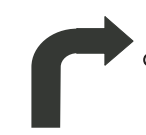

EVALUATE

practice

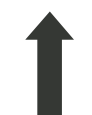

IMPLEMENT practice change

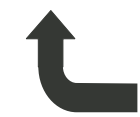

ASSESS need for

DESIGN change in practice

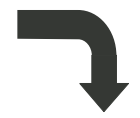

ASK an answerable research question

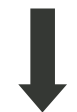

SYNTHESISE best evidence

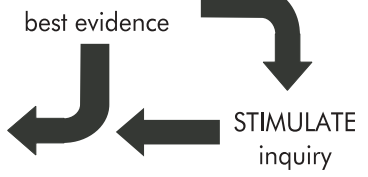

Adapted from Rosswurm ML and Larrabee JH. Image J Nurs Sch 1999; 31: 317-22.

\section{Prioritising and disseminating research findings to} healthcare programmes

The limited number of HHS nurses with critical appraisal knowledge and skills, combined with the clinical demands of providing direct care, mitigate against nurses participating in EBP projects to search for, appraise, disseminate, and apply relevant high quality research. With these barriers in mind, the Firstly, the linear framework was re-oriented to a feedback loop change in practice 
EBN Committee decided to develop a system to identify, rate, and disseminate important research findings to healthcare programmes in the organisation.

Recognising the journal $E B N$ as a powerful tool in harnessing EBP, especially during the early development of the concept at HHS, the EBN Committee developed a project to determine the usefulness and feasibility of recommending research findings abstracted in $E B N$ to relevant HHS healthcare programmes.

As a first step, an $E B N$ abstract rating form was developed to rate specific $E B N$ abstracts of empirical research and overviews from the latest $E B N$ issue. The rating form identified the $E B N$ citation, the citation for the original research article, and the major finding, as stated in the declarative title of the $E B N$ abstract. A series of checklists followed. Firstly, the raters (EBN Committee members) indicated whether the research was primarily applicable to clinical care or some aspect of healthcare administration and specified the HHS programmes to which the findings would likely be relevant. Raters then checked off a series of factors that might increase or decrease perceived importance of disseminating the research findings, including the magnitude of the health problem, the burden of illness, and the extent of variation in clinical practice. Finally, raters assigned a global rating of the priority for dissemination on a scale of $1-7$, where 7 was the highest priority and 1 was the lowest priority. Raters were also asked to indicate their confidence in assigning the rating. A glossary of terms and examples was developed.

The Committee refined the rating form during several iterations, and then $7 E B N$ quantitative research abstracts were randomly selected for rating. 6 EBN Committee members independently reviewed each abstract and commentary before completing the rating form. Raters completed a single review in $\leq 20$ minutes. 3 of the 7 abstracts received strong mean global ratings for implementation (6-7 points) and 4 received moderate mean global ratings (4-5 points). Global ratings had moderate inter-rater reliability $\left(\mathrm{r}_{\mathrm{ICC}}=0.60\right)$. The Committee decided to disseminate the findings and accompanying information for abstracts that received an average global rating of $\geq 5$ by $\geq 3$ raters.

In a pretest of the dissemination of research findings abstracted in $E B N$, programme managers and educators were receptive to receiving the abstracts, and most provided specific details about how the information could be used in clinical practice. However, at this time, it is not known whether the managers or their delegates have implemented the specific findings. Because the process of rating, recommending, and disseminating study abstracts and commentaries from $E B N$ appears viable, this project is continuing. 1 year follow up evaluation is in process for programme and/or unit clinical decision making regarding the major findings cited in the $E B N$ abstractions.

\section{Evaluating an important EBP application in direct patient care}

During the process of reviewing $E B N$ abstracts, the EBN Committee identified a study that concluded that chlorhexidine was a more effective skin antiseptic than 2 other cleansing agents in preventing probable peripheral intravenous catheter related infection. ${ }^{16}$ This resonated with EBN Committee members because confusion existed among the members as to which of the 2 cleansing agents, chlorhexidine or alcohol, was required at HHS for this purpose. Lack of clarity was borne out when the clinical policy was consulted. Although the approved policy for skin cleansing before intravenous initiation was changed to chlorhexidine in 2000 by a regional infection control committee, the written policy statement was non-specific, stating only that the "recommended" cleansing agent should be used, without naming chlorhexidine explicitly.

This clinical issue presented an excellent example for the evaluation of adherence to EBP, specifically as it relates to the "integrate and maintain the change in practice" step in the nursing EBP model (see figure 1). Firstly, the effectiveness of chlorhexidine was clearly established, showing consistency across a number of studies. In fact, Mermel published a systematic overview indicating the need to change practice to the use of chlorhexidine based on Level IIa evidence from randomised trials in 2000. ${ }^{17}$ Secondly, the issue is relevant, as most intravenous therapy is initiated by nurses. Thirdly, morbidity and mortality associated with intravenous catheter infections made this a critical patient safety issue. Furthermore, because chlorhexidine swabs are more costly than alcohol swabs, the appropriate use of chlorhexidine swabs is an interprofessional cost containment issue with implications for hospital departments, such as stores. Finally, examination of this clinical EBP issue presented an opportunity for interactive learning involving HHS nursing staff. ${ }^{5}$

With these reasons in mind, a 1 day census survey was conducted by the EBN Committee involving all Registered Nurses and Registered Practical Nurses (excluding those without any of the skills in question). A short, pretested, closed ended questionnaire was administered by the EBN Committee members or their delegates.

324 HHS nurses participated, yielding a response rate $>90 \%$. The survey findings indicated that $<75 \%$ of nurses reported using chlorhexidine (the proper agent for asepsis before intravenous initiation), whereas $42 \%$ reported using chlorhexidine for asepsis before venipuncture (where alcohol was the prescribed aseptic agent).

This exercise led to a number of interventions designed to change practice. An explicit policy statement about the cleansing agent to be used for peripheral intravenous cannulation was disseminated. A one page description of the study and its results was distributed as feedback to all clinical unit nursing staff. The EBN Committee members acted as champions within their clinical areas, doing one on one teaching with staff. Advance practice nurses (APNs) and nurse educators were asked to incorporate appropriate chlorhexidine use into educational strategies. In areas where large numbers of intravenous cannulations were initiated, unit specific survey results were provided. Furthermore, a poster presentation of the study was included at the annual HHS nursing conference.

In keeping with managerial responsibilities for effecting change and ensuring implementation of high quality, up to date research in practice, the clinical managers were asked to identify, and reduce or eliminate, barriers to appropriate chlorhexidine use on their units. ${ }^{18}$ In areas identified as having low adherence to appropriate chlorhexidine use, a diagrammatic prop illustrating "best practice" was placed above the alcohol and chlorhexidine swab containers. A follow up survey will be done 12 months after implementation of the last strategy to effect change, and clinical managers will be asked to identify unit specific changes initiated to increase adherence. ${ }^{18}$

\section{Other progress and plans}

At present, the EBN Committee has been mandated to oversee the implementation of nursing related clinical practice guidelines, such as the Registered Nurses Association of Ontario (RNAO) Best Practice Guidelines, which focus on the prevention of falls and skin breakdown. ${ }^{19-21}$

Seasoned nursing staff with credibility among peers will be sought to participate as members on the EBN Committee. Small 
group problem based workshops on effective literature searching and critical appraisal are being planned, and independent learning formats will be sought as adjuncts to other education on this topic.

Educational EBN Committee "Lunch and Learn" sessions are being continued for nurses in various HHS health programmes. Nurse educators and APNs are being encouraged to become members of the Committee, and senior nursing students will continue to participate in EBN Committee work as part of their research electives. ${ }^{22}$

\section{Concluding remarks}

The rapidity of change and the reorganisation of nursing services within the healthcare sector present challenges for the advancement of EBP. Managers and administrators should facilitate the uptake of practice based on current, high quality research by formalising the expectation that nursing care be evidence-based. ${ }^{18}$ Healthcare and professional organisations must pursue systems that facilitate easy access to research evidence and educational EBP materials. Researchers need to assume more responsibility for EBP by helping clinicians implement current research findings. ${ }^{23}$

This paper illustrates one tertiary hospital's evolving experience with the implementation of EBP in nursing. The EBN Committee strives to respond to the needs and capabilities of the nurses and the organisation, apply important principles for closing the research gap, and use systematic and varied approaches to create change in practice.

E. ANN MOHIDE, RN, MHSc, MSc Associate Professor, School of Nursing, McMaster University, Co-Chair, Evidence-Based Nursing (EBN) Committee Hamilton Health Sciences Hamilton, Ontario, Canada BERNICE KING, RN, MHSc Clinical Nurse Specialist, Professional Affairs Clinical Faculty, School of Nursing, McMaster University
Co-Chair, Evidence-Based Nursing Committee Hamilton Health Sciences Hamilton, Ontario, Canada The authors are grateful to the EBN Committee members for their continuing commitment,
vision, and efforts to implement EBP. Thanks are extended to the BScN students who contributed to these activities: Maureen O'Neil, Loretta O'Neill, Emily Booth, Katie King, Grace Sa, Erin Stockwell, Heather Donaldson-Mulgrove and Shelley Harrison.

1 Funk SG, Tornquist EM, Champagne M. Barriers and facilitators of research utilization. An integrative review. Nurs Clin North Am 1995;30: 395-407.

2 Royle J, Blythe J. Promoting research utilisation in nursing: the role of the individual, organisation, and environment. Evid Based Nurs 1998;1:71-2.

3 Estabrooks CA. Will evidence-based nursing practice make practice perfect? Can J Nurs Res 1998;30:15-36.

4 Newman M, Papadopoulos I. Developing organisational systems and culture to support evidence-based practice: the experience of the EvidenceBased Ward Project. Evid Based Nurs 2000;3:103-5.

5 Moulding NT, Silagy CA, Weller DP. A framework for effective management of change in clinical practice: dissemination and implementation of clinical practice guidelines. Qual Health Care 1999;8:177-83.

6 DiCenso A, Cullum N, Ciliska D. Evidence-Based Nursing: 4 years down the road. Evid Based Nurs 2002;5:4-5.

7 Ciliska D, DiCenso A, Cullum N. Centres of evidence-based nursing: directions and challenges. Evid Based Nurs 1999;2:102-4.

8 Rosswurm MA, Larrabee JH. A model for change to evidence-based practice. Image J Nurs Sch 1999;31:317-22.

9 Van Mullem C, Burke LJ, Dohmeyer K, et al. Strategic planning for research use in nursing practice. J Nurs Adm 1999;29:38-45.

10 Stetler CB. Refinement of the Stetler/Marram model for application of research findings to practice. Nurs Outlook 1994;42:15-25.

11 Titler MG, Kleiber C, Steelman V, et al. Infusing research into practice to promote quality care. Nurs Res 1994;43:307-13.

12 Barnsteiner JH, Ford N, Howe C. Research utilization in a metropolitan children's hospital. Nurs Clin North Am 1995;30:447-55.

13 Pearcey P, Draper P. Using the diffusion of innovation model to influence practice: a case study. J Adv Nurs 1996;23:714-21.

14 Warren JJ, Heermann JA. The Research Nurse Intern program. A model for research dissemination and utilization. J Nurs Adm 1998;28:39-45.

15 Hunt JM. Barriers to research utilization. J Adv Nurs 1996;23:423-5.

16 LeBlanc A, Cobbett S. Traditional practice versus evidence-based practice for IV skin preparation. Can J Infect Control 2000;Spring:9-14.

17 Mermel LA. Prevention of intravascular catheter-related infections. Ann Intern Med 2000;132:391-402.

18 Browman GP, Snider A, Ellis P. Negotiating for change. The healthcare manager as catalyst for evidence-based practice: changing the healthcare environment and sharing experience. Healthc Pap 2002;3:10-22.

19 Kearsey K. Launch of nursing Best Practice Guidelines: envisioned as next step to more effective patient care. Registered Nurse 2001;November/ December:8-11.

20 DiCenso A, Virani T, Bajnok I, et al. A toolkit to facilitate the implementation of clinical practice guidelines in healthcare settings. Hosp $Q$ 2002; 5:55-60.

21 Registered Nurses Association of Ontario (RNAO). http://www.rnao.org.

22 Fonteyn M. Print and online versions of Evidence-Based Nursing: innovative Fonteyn M. Print and online versions of Evidence-Based Nursing:

23 Waddell C. So much research evidence, so little dissemination and uptake: mixing the useful with the pleasing. Evid Based Nurs 2002;5:38-40. 